\title{
DISTRIBUTIONS WITH COMPLEX STRUCTURE
}

\author{
DEDICATED TO PROFESSOR TOMINOSUKE OTSUKI \\ ON HIS SIXTIETH BIRTHDAY
}

\author{
BY SHIGERU ISHIHARA
}

Any real hypersurface $M$ immersed in a complex manifold admits a hyperdistribution with complex structure induced from the complex structure of the ambient manifold. On the other hand it is well known that $M$ is endowed with a pseudo-conformal structure under some restrictions (See Cartan [1], Chern and Moser [2], Tanaka [7]). In the present paper we shall study hyperdistribution with complex structure in a manifold of odd dimensions and show that a hyperdistribution with complex structure determines a pseudo-conformal structure if it is torsionless and non-degenerate.

First of all, definitions and some properties of almost contact structures will be recalled in $\S 1$ for later use. $\S 2$ will be devoted to make clear relationships between a hyperdistribution $D$ with complex structure and almost contact structures associated with $D$. We shall prove in $\S 3$ some necessary and sufficient conditions for $D$ to be torsionless. In $\S 4$ we shall define pseudo-conformal mappings, transformations and infinitesimal pseudo-conformal transformations and obtain some of their properties.

\section{$\S 1$. Almost contact structures.}

In this section, we shall recall definitions and some properties of almost contact structures for later use. Let $M$ be a differentiable manifold of dimension $2 n+1(\geqq 3)$ and there be given in $M$ a tensor field $f$ of type $(1,1)$, a vector field $\xi$ and a 1 -form $\theta$ satisfying

$$
f^{2}=-I+\theta \otimes \xi, \quad f \xi=0, \quad f \circ \theta=0, \quad \theta(\xi)=1,
$$

where $I$ denotes the identity tensor field of type $(1,1)$ in $M_{*}^{*}$ Then such a triple $(f, \xi, \theta)$ is called an almost contact structure. We define now tensor fields $S$ of type $(1,2), T$ of type $(0,2), P$ of type $(1,1)$ and $Q$ of type $(0,1)$ respectively by

Received December 15, 1976.

*) Manifolds, tensor fields, geometric objects and mappings we consider are assume to be differentiable and of class $C^{\infty}$ unless otherwise stated. 


$$
S(X, Y)=[f X, f Y]-f[f X, Y]-f[X, f Y]+f^{2}[X, Y]+d \theta(X, Y) \xi,
$$

$$
\begin{gathered}
T(X, Y)=d \theta(f X, Y)-d \theta(f Y, X), \\
P=-L_{\xi} f, \quad Q=-L_{\xi} \theta,
\end{gathered}
$$

where $L_{Z}$ denotes the operator of Lie derivation with respect to a vector field $Z$. $^{*}$ The tensor field $S$ thus defined is called the torsion tensor of the almost contact structure $(f, \xi, \theta)$. The tensor field $G$ of type $(0,2)$ defined by

$$
G(X, Y)=d \theta(f X, Y)
$$

is called the Levi tensor of $(f, \xi, \theta)$. Then (1.2) implies

$$
T(X, Y)=G(X, Y)-G(Y, X) \text {. }
$$

There are well known identities

$$
\begin{gathered}
T(X, Y)=\theta(S(f X, \xi) \theta(Y)-S(X, f Y)), \\
P(X)=-S(f X, \xi)
\end{gathered}
$$

(See Sasaki [5], p. 5-2, for example). The following propositions $\left(\mathrm{P}_{1}\right)$ and $\left(\mathrm{P}_{2}\right)$ are also well known (See Sasaki [5], p. 5-6, for example):

$$
\begin{aligned}
& \left(\mathrm{P}_{1}\right) \quad S=0 \text { implies } T=0, \quad P=0 \text { and } Q=0 ; \\
& \left(\mathrm{P}_{2}\right) \quad P=0 \text { implies } Q=0 .
\end{aligned}
$$

When the condition $S=0$ is satisfied, the almost contact structure $(f, \xi, \theta)$ is usually said to be normal.

Denote by $f_{\imath}{ }^{h}, \xi^{h}$ and $\theta_{\imath}$ respectively components of $f, \xi$ and $\theta$ in each coordinate neighborhood $U$ of $M *^{* *}$ ) Then the Levi tensor $G$ has components of the form

$$
G_{j i}=f_{j}{ }^{k}\left(\partial_{k} \theta_{\imath}-\partial_{i} \theta_{k}\right)
$$

and the tensor fields $S$ and $T$ have respectively components of the form

$$
\begin{gathered}
S_{j i}{ }^{h}=f_{\jmath}{ }^{k} \partial_{k} f_{\imath}{ }^{h}-f_{\imath}{ }^{k} \partial_{k} f_{\jmath}{ }^{h}-\left(\partial_{\jmath} f_{\imath}{ }^{k}-\partial_{\imath} f_{\jmath}{ }^{k}\right) f_{k}{ }^{h}+\left(\partial_{\jmath} \theta_{\imath}-\partial_{i} \theta_{\jmath}\right) \xi^{h}, \\
T_{j \imath}=G_{j i}-G_{\imath \jmath}
\end{gathered}
$$

with respect to local coordinates $\left(x^{h}\right)$, where $\partial_{\imath}=\partial / \partial x^{2}$.

*) $X, Y, Z, \cdots \cdots$ denote arbitrary differentiable vector fields in $M$ unless otherwise stated.

**) The indices $h, i, \jmath, k, l$ run over the rang $\{1, \cdots, 2 n+1\}$ and the summation convension will be used with respect to this system of indices. 


\section{$\S 2$. Distributions with complex structure and almost contact structures.}

Let there be given a distribution $D$ of dimension $2 n$ in a manifold of dimension $2 n+1(\geqq 3)$. Suppose there is given a linear transformation $J_{P}: D_{P} \rightarrow$ $D_{P}$ for each point $P$ of $M, D_{P}$ being the value of $D$ at $P$, in such a way that $J_{P}{ }^{2} v=-v$ for any vector $v$ belonging to $D_{P}$. Moreover assume that for any differentiable vector field $X$ belonging to $D$ the vector field $J X$ defined by the correspondence $P \mapsto J_{P} X_{P}, X_{P}$ being the value of $X$ at $P$, is also differentiable. Thus we have a tensor field $J$ of type $(1,1)$ in the distribution $D$ if we define $J$ by the correspondence $J: X \rightarrow J X, X$ being an arbitrary vector field belonging to $D$. Such a tensor field defined in $D$ is called a complex structure in $D$. The $J$ just defined in $D$ satisfies $J^{2}=-I_{D^{\prime}}$ where $I_{D}$ denotes the identity tensor field of type $(1,1)$ in $D$. The pair $(D, J)$ or, simply saying, $D$ is called a hyperdistribution with complex structure $J$. In the present section, the manifold $M$ of dimension $2 n+1$ is assumed to admit a hyperdistribution $D$ with complex structure $J$.

Take a vector field $\xi$ in a coordinate neighborhood $U$ of $M$ in such a way that $\theta(\xi)=1$, where $D$ is defined by $\theta=0$ in $U$. Then we can define in $U$ a tensor field $f$ of type $(1,1)$ by

$$
f X=J(X-\theta(X) \xi)
$$

because $X-\theta(X) \xi$ belongs to $D$, i. e., because $\theta(X-\theta(X) \xi)=0$. Thus we have an almost contact structure $(f, \xi, \theta)$ in $U$, which will be called an almost contact structure associated with $D$ in $U$.

When the manifold $M$ admitting a hyperdistribution $D$ with complex structure $J$ is orientable, there are a global 1-form $\theta$ and a global vector field $\xi$ such that $D$ is defined by $\theta=0$ and $\theta(\xi)=1$. Thus in such a case there is a global almost contact structure $(f, \xi, \theta)$ associated with $D$.

Comming back to the general situation, we put in $U \tilde{\theta}=\alpha \theta$ with non-vanishing function $\alpha$ defined in $U$ and take a vector field $\tilde{\xi}$ in $U$ such that $\tilde{\theta}(\tilde{\xi})=1$. Then we find $\tilde{\xi}=\alpha^{-1}(\xi-f A)$, where $A$ is a vector field in $U$ such that $\theta(A)=0$, i. e., such that $A$ belongs to $D$. Thus there is in $U$ another almost contact structure $(\tilde{f}, \tilde{\xi}, \tilde{\theta})$ associated with $D$, which is defined by

$$
\tilde{f}=f-\theta \otimes A, \quad \tilde{\xi}=\frac{1}{\alpha}(\xi-f A), \quad \tilde{\theta}=\alpha \theta .
$$

We call (2.2) the equations of change of almost contact structures associated with $D$. In such a case the two almost contact structures $(f, \xi, \theta)$ and $(\tilde{f}, \xi, \tilde{\theta})$ are said to be equivalent to each other. Any property $(P)$ of $(f, \xi, \theta)$ associated with $D$ can be considered to endow $D$ with a property if $(P)$ is invariant under the change (2.2) of associated almost contact structures. 
Denote by $S, T$ and $\tilde{S}, \tilde{T}$ the tensor fields defined by (1.2) respectively in terms of $(f, \xi, \theta)$ and $(\tilde{f}, \tilde{\xi}, \tilde{\theta})$, where $(f, \xi, \theta)$ and $(\tilde{f}, \tilde{\xi}, \tilde{\theta})$ are related to each other by the change (2.2). Then, using (1.8) and (2.2), we can easily verify

$$
\begin{gathered}
\widetilde{S}_{j i}{ }^{h}=S_{j i}{ }^{h}-T_{j i} A^{h}-\theta_{j}\left[A^{h} L_{A} \theta_{\imath}+L_{A} f_{\imath}{ }^{h}+\alpha_{i}\left(\xi^{h}-f_{k}{ }^{h} A^{k}\right)\right] \\
+\theta_{\imath}\left[A^{h} L_{A} \theta_{j}+L_{A} f_{\jmath}{ }^{h}+\alpha_{j}\left(\xi^{h}-f_{k}{ }^{h} A^{k}\right)\right], \\
\widetilde{T}_{j i}=\alpha\left[T_{j i}-\theta_{j}\left(L_{A} \theta_{i}+f_{\imath}{ }^{k} \alpha_{k}\right)+\theta_{\imath}\left(L_{A} \theta_{\jmath}+f_{\jmath}{ }^{k} \alpha_{k}\right)\right],
\end{gathered}
$$

where $\widetilde{S}_{j i}{ }^{h}$ and $\tilde{T}_{j i}$ are components of $\tilde{S}$ and $\tilde{T}$ respectively, $A^{h}$ are components of $A$ and $\alpha_{\imath}$ are defined by $\alpha_{i}=\alpha^{-1} \partial_{i} \alpha$. We note here that $\theta(A)=\theta_{\imath} A^{2}=0$ is satisfied. Let $G$ and $\tilde{G}$ be the Levi tensors of $(f, \xi, \theta)$ and $(\tilde{f}, \tilde{\xi}, \tilde{\theta})$ respectively. Then, using (1.7) and (2.2), we obtain

$$
\tilde{G}_{j \imath}=\alpha\left[G_{j i}+f_{\jmath}^{k} \alpha_{k} \theta_{i}-\theta_{\jmath} A^{k}\left(\partial_{k} \theta_{\imath}-\partial_{i} \theta_{k}\right)-\left(A^{k} \alpha_{k}\right) \theta_{j} \theta_{\imath}\right]
$$

where $G_{j i}$ and $\tilde{G}_{j i}$ are components of $G$ and $\tilde{G}$ respectively.

Consider a tensor field $K$ (local or global) of type $(1,2)$ (resp. of type $(0,2)$ ) in $M$. If $K(X, Y)=0$ for any vector fields $X$ and $Y$ belonging to $D$, then we denote this fact by $K \equiv 0,(\bmod \theta)$. Let $K_{j i}{ }^{h}$ (resp. $\left.K_{j i}\right)$ be components of $K$. Then the condition $K \equiv 0,(\bmod \theta)$ is represented by $K_{j 2}{ }^{h}=\theta_{0} A_{2}{ }^{h}+\theta_{\imath} B_{j}{ }^{h}$ (resp. $K_{j i}=\theta_{j} A_{\imath}+\theta_{\imath} B_{j}$ ), where $A_{\imath}{ }^{h}$ and $B_{\imath}{ }^{h}$ (resp. $A_{\imath}$ and $B_{\imath}$ ) are respectively components of certain tensor fields. Thus the condition $K \equiv 0,(\bmod \theta)$ is equivalently represented by $K_{j i}{ }^{h} \equiv 0,\left(\bmod \theta_{\jmath}, \theta_{\imath}\right)$ (resp. $\left.K_{j i} \equiv 0,\left(\bmod \theta_{\jmath}, \theta_{\imath}\right)\right)$ in terms of components. If two tensor fields $K$ and $L$ of type $(1,2)$ (resp. of type $(0,2)$ ) satisfy the condition $K-L \equiv 0,(\bmod \theta)$, then we denote this fact by $K \equiv L,(\bmod \theta)$. Using such a kind of notations, we see that (2.5) reduces to

$$
\tilde{G} \equiv \alpha G(\bmod \theta) \text {. }
$$

with non-vanishing function $\alpha$. We now have from (2.6)

Proposition 2.1. Let $D$ be a hyperdistribution with complex structure in $M$. Denote by $G_{D}$ the restruction of the Levi tensor $G$ to $D$, where $G$ is given by (1.3) in terms of an $(f, \xi, \theta)$ associated with $D$. Then $G_{D}$ is unzquely determined up to a non-vanishing factor $\alpha$ at each point $P$ of $M$.

By means of Proposition 2.1, the rank $r$ of $G_{D}$ at each point $P$ of $M$ is independent of the choice of $(f, \xi, \theta)$ associated with $D$. When $r$ is a constant independent of points of $M$, the $D$ is said to be of rank $r$, where $r$ is necessarily even. The $G_{D}$ or, roughly speaking, $G$ is called the Levi tensor of $D$. When $G_{D}$ is of maximum rank $2 n$, the hyperdistribution $D$ is said to be non-degenerate.

Next, the condition $S \equiv 0,(\bmod \theta)$ implies the condition $T \equiv 0,(\bmod \theta)$ by means of (1.5). Thus, using (2.3) and (2.4), we see easily that the condition $S \equiv 0,(\bmod \theta)$ is equivalent to the condition $\widetilde{S} \equiv 0,(\bmod \theta)$. Hence we now have 
Proposition 2.2. The condition $S \equiv 0,(\bmod \theta)$ for an almost contact structure $(f, \xi, \theta)$ associated with a hyperdistribution $D$ with complex structure is left invariant by the change (2.2) of associated almost contact structures.

Proposition 2.2 means that the condition $S \equiv 0,(\bmod \theta)$ for an almost contact structure $(f, \xi, \theta)$ associated with $D$ gives to $D$ itself a property. Thus, if $S \equiv 0$, $(\bmod \theta)$ is satisfied at each point for an associated almost contact structure $(f, \xi, \theta)$, then $D$ is said to be torszonless. Therefore, if there is at least one almost contact structure associated with $D$ which is normal, then $D$ is necessarily torsionless.

Proposition 2.3. If a hyperdistribution $D$ with complex structure $J$ is torsionless, then the Levi tensor $G$ of $D$ satisfies

$$
G_{j i} \equiv G_{i,} \bmod \left(\theta_{\jmath}, \theta_{\imath}\right)
$$

and

$$
G(J X, J Y) \equiv G(X, Y)
$$

for any vector fields $X$ and $Y$ belonging to $D$.

Proof. Since $S \equiv 0,(\bmod \theta)$ implies $T \equiv 0,(\bmod \theta)$, equations $(1.2),(1.3)$ and (1. 8) imply Proposition 2.3.

Proposition 2.3 shows that for a torsionless hyperdistribution $D$ with complex structure $J$ its Levi tensor $G_{D}$ is a symmetric tensor field of type $(0,2)$ in $D$ and Hermitian with respect to $J$. We now state a lemma for later use.

LEMMA 2.4. If $S \equiv 0,(\bmod \theta)$ and $P=0$ are satisfied for an almost contact structure $(f, \xi, \theta)$, then $S=0$, i.e., $(f, \xi, \theta)$ is normal.

Proof. Since $S \equiv 0,(\bmod \theta)$, we can put $S_{j i}{ }^{h}=\theta_{\jmath} A_{\imath}{ }^{h}-\theta_{\imath} A_{\jmath}{ }^{h}$. On the otherhand, since $P=0$, we have from (1.6) $S(f X, \xi)=0$. Thus substituting $S_{j i}{ }^{h}=$ $\theta_{\jmath} A_{\imath}{ }^{h}-\theta_{\imath} A_{\jmath}{ }^{h}$ into this equation gives $A_{k}{ }^{h} f_{\jmath}{ }^{k}=0$, which implies $A_{\jmath}{ }^{h}=\theta_{\jmath} A_{k}{ }^{h} \xi^{k}$. Therefore we obtain $S_{j i}{ }^{h}=0$, i. e., $S=0$.

\section{§3. Adapted frames and coframes.}

Let there be given a hyperdistribution $D$ with complex structure $J$ in a manifold $M$ of dimension $2 n+1$. Consider an almost contact structure $(f, \xi, \theta)$ associated with $D$ in each coordinate neighborhood $U$ of $M$. Let $\left\{\xi_{b}, \xi\right\}$ be a field of affine frames in $U$ such that each $\xi_{b}$ belongs to $D$.*) Since $f \xi_{b}=J \xi_{b}$ belongs to $D$, we can put

$$
f \xi_{b}=J_{b}{ }^{a} \xi_{a},
$$

*) The indices $a, b, c, d, e$ run over the range $\{1, \cdots, 2 n\}$ and the summation convension will be used with respect to this system of indices. 
where $J_{b}{ }^{a}$ satisfies

$$
J_{e}^{a} J_{b}^{e}=\delta_{b}^{a}
$$

because $f^{2} \xi_{b}=J^{2} \xi_{b}=-\xi_{b}$. If the frame $\left\{\xi_{b}, \xi\right\}$ is suitably taken, the matrix $\left(J_{b}{ }^{a}\right)$ has the following numerical value:

$$
\left(J_{b}{ }^{a}\right)=\left(\begin{array}{cc}
0 & -E \\
E & 0
\end{array}\right)
$$

where $E$ denotes the $(n, n)$ unit matrix. Such an affine frame $\left\{\xi_{b}, \xi\right\}$ is said to be adapted to $D$, if $\left(J_{b}{ }^{a}\right)$ has the value given by (3.2) with respect to $\left\{\xi_{b}, \xi\right\}$.

Let $\left\{\xi_{b}, \xi\right\}$ be a frame adapted to $D$ in $U$. Then components of the Levi tensor $G_{D}$ with respect to $\left\{\xi_{b}, \xi\right\}$ are given by

$$
G_{c b}=G_{D}\left(\xi_{c}, \xi_{b}\right)=G\left(\xi_{c}, \xi_{b}\right) .
$$

If we now put

$$
J_{c b}=d \theta\left(\xi_{c}, \xi_{b}\right)
$$

then we find

$$
J_{c b}=J_{c}^{e} G_{e b}
$$

because of $G_{j i}=f_{j}{ }^{k}\left(\partial_{k} \theta_{i}-\partial_{i} \theta_{k}\right)$ which appeared in (1.7).

We take another frame $\left\{\tilde{\xi}_{b}, \tilde{\xi}\right\}$ adapted to $D$ in another coordinate neighborhood $\tilde{U}$ of $M$. Then, using (2.2), we obtain in $U \cap \tilde{U}$

$$
\tilde{\xi}=\frac{1}{\alpha}(\xi-f A), \quad \tilde{\xi}_{b}=U_{b}{ }^{a} \xi_{a},
$$

where $\theta(A)=0$ and the matrix $\left(U_{b}^{a}\right)$ belongs to the real respresentation of the complex linear group $C L(n)$, because

$$
J_{e}^{a} U_{b}{ }^{e}=U_{e}{ }^{a} J_{b}{ }^{e} .
$$

Thus the structure group of the tangent bundle of $M$ is, as is well known, reducible to the group of all matrices of the form

$$
\left(\begin{array}{cc}
\alpha & 0 \\
B^{a} & U_{b}{ }^{a}
\end{array}\right)
$$

where $\alpha$ is non-zero and $\left(U_{b}{ }^{a}\right)$ belongs to the real representation of $C L(n)$.

The equations (2.2), (2.6) and (3.6) imply that

$$
\tilde{G}_{c b}=\alpha U_{c}^{e} U_{b}^{d} G_{e d}
$$

is established in $U \cap \tilde{U}, \tilde{G}_{c b}$ being components of the Levi tensor $\tilde{G}_{D}$ with respect to $\left\{\tilde{\xi}_{b}, \tilde{\xi}\right\}$. 
Given a frame $\left\{\xi_{b}, \xi\right\}$ adapted to $D$ in $U$, we denote by $\left\{\theta^{a}, \theta\right\}$ the coframe dual to $\left\{\xi_{b}, \xi\right\}$ in $U$. That is to say,

$$
\theta^{a}\left(\xi_{b}\right)=\delta_{b}^{a}, \quad \theta^{a}(\xi)=0, \quad \theta\left(\xi_{b}\right)=0, \quad \theta(\xi)=1 .
$$

Such a coframe $\left\{\theta^{a}, \theta\right\}$ is said to be adapted to $D$ in $U$. We now have

THEOREM 3.1. Let $D$ be a hyperdistribution with complex structure $J$ in a manifold $M$ of dimension $2 n+1(\geqq 3)$. Then $D$ is torsionless if and only if in each coordinate neighborhood $U$ of $M$ any coframe $\left\{\theta^{a}, \theta\right\}$ adapted to $D$ satisfies the following equations:

$$
\begin{aligned}
d \theta & =\frac{1}{2} J_{c b} \theta^{c} \wedge \theta^{b}+\theta \wedge \phi, \\
d \theta^{a} & =\frac{1}{2}{\phi_{c b}}^{a} \theta^{c} \wedge \theta^{b}+\theta \wedge \phi^{a},
\end{aligned}
$$

$\phi$ and $\phi^{a}$ being certain 1 -forms in $U$, where

$$
\begin{array}{ll}
J_{c b}=-J_{b c}, & J_{c b}=J_{c}^{e} G_{e b}, \\
G_{c b}=G_{b c}, & G_{c b}-J_{c}^{e} J_{b}{ }^{d} G_{e d}=0
\end{array}
$$

(i.e., $G_{c b}$ and $J_{c b}$ are hybred with respect to $J_{b}{ }^{a}$ ) and, where

$$
\begin{aligned}
& \phi_{c b}{ }^{a}=-\phi_{b c}{ }^{a}, \\
& \phi_{c b}{ }^{a}-J_{c}^{e} J_{b}{ }^{a} \phi_{c d}{ }^{a}=0, \quad \phi_{c b}{ }^{a}+J_{b}^{e} J_{d}{ }^{a} \phi_{c e}{ }^{d}=0
\end{aligned}
$$

(i.e., $\phi_{c b}{ }^{a}$ is hybrid with respect to indices $c, b$ and pure with respect to indices $a, b)$.

Proof. The given $D$ is torsionless if and only if $S \equiv 0,(\bmod \theta)$, i. e., if and only if $S\left(\xi_{c}, \xi_{b}\right)=0$, which is equivalent to

$$
\left[f \xi_{c}, f \xi_{b}\right]-f\left[f \xi_{c}, \xi_{b}\right]-f\left[\xi_{c}, f \xi_{b}\right]+f^{2}\left[\xi_{c}, \xi_{b}\right]+d \theta\left(\xi_{c}, \xi_{b}\right)=0
$$

or equivalently to

$$
\begin{gathered}
\theta\left(\left[f \xi_{c}, f \xi_{b}\right]\right)+d \theta\left(\xi_{c}, \xi_{b}\right)=0, \\
\theta^{a}\left(\left[f \xi_{c}, f \xi_{b}\right]-f\left[f \xi_{c}, \xi_{b}\right]-f\left[\xi_{c}, f \xi_{b}\right]+f^{2}\left[\xi_{c}, \xi_{b}\right]\right)=0 .
\end{gathered}
$$

Since $f \xi_{b}=J_{b}{ }^{a} \xi_{a},(3.14)$ is equivalent to

$$
\begin{gathered}
J_{c}^{e} J_{b}{ }^{d} d \theta\left(\xi_{e}, \xi_{d}\right)=d \theta\left(\xi_{c}, \xi_{b}\right), \\
J_{c}^{e} J_{b}{ }^{d} d \theta^{a}\left(\xi_{e}, \xi_{d}\right)-J_{c}^{e} J_{d}^{a} d \theta^{d}\left(\xi_{e}, \xi_{b}\right) \\
-J_{b}^{e} J_{d}^{a} d \theta^{d}\left(\xi_{c}, \xi_{e}\right)-d \theta^{a}\left(\xi_{c}, \xi_{b}\right)=0 .
\end{gathered}
$$


If we now put

$$
J_{c b}=d \theta\left(\xi_{c}, \xi_{b}\right), \quad \phi_{c b}^{a}=d \theta^{a}\left(\xi_{c}, \xi_{b}\right),
$$

then we see that (3.15) is equivalent to

$$
\begin{aligned}
& J_{c b}-J_{c}^{e} J_{b}{ }^{d} J_{e d}=0, \\
& A_{c b}{ }^{a}+J_{b}^{e} J_{d}{ }^{a} A_{c e}{ }^{d}=0, \text { where } A_{c b}{ }^{a}=\phi_{c b}{ }^{a}-J_{c}{ }^{e} J_{b}{ }^{d} \phi_{e d}{ }^{a} .
\end{aligned}
$$

The equations (3.16) is equivalent to (3.11). Moreover the first and the second equations of (3.17) are equivalent to (3.12) and (3.13) respectively. Thus Theorem 3.1 is proved completely.

Next we have

THEOREM 3.2. Let $D$ be a hyperdistribution with complex structure $J$ is a manifold $M$ of dimension $2 n+1(\geqq 3)$. A frame $\left\{\xi_{b}, \xi\right\}$ adapted to $D$ is normal, i.e., an almost contact structure $(f, \xi, \theta)$ associated with $D$ is normal in a coordinate nerghborhood $U$ of $M$, if and only if its dual coframe $\left\{\theta^{a}, \theta\right\}$ satisfies

$$
d \theta=\frac{1}{2} J_{c b} \theta^{c} \wedge \theta^{b}
$$

$$
d \theta^{a}=\frac{1}{2}{\phi_{c b}}^{a} \theta^{c} \wedge \theta^{b}+{\phi_{b}}^{a} \theta \wedge \theta^{b},
$$

where $J_{c b}$ and ${\phi_{c b}}^{a}$ satısfy (3.12) and (3.13) respectively and, where ${\phi_{b}}^{a}$ satısfies

$$
\phi_{b}{ }^{a}+J_{b}{ }^{e} J_{d}{ }^{a} \phi_{e}{ }^{d}=0
$$

(i.e., $\phi_{b}{ }^{a}$ is pure with respect to $J_{b}{ }^{a}$ ).

Proof. A frame $\left\{\xi_{b}, \xi\right\}$ adapted to $D$, i. e., an almost contact structure $(f, \xi, \theta)$ associated with $D$ is normal if and only if $S=0$, which is equivalent to

$$
S\left(\xi_{c}, \xi_{b}\right)=0 \text { and } S\left(\xi_{c}, \xi\right)=0 .
$$

The first equation of (3.20) is equivalent to the conclusions (3.11), (3.12) and (3.13) stated in Theorem 3.1 and the second one of (3.20) is equivalent to

$$
\begin{gathered}
\theta^{a}\left(S\left(\xi_{c}, \xi\right)\right)=-\theta^{a}\left(f\left[f \xi_{c}, \xi\right]\right)+\theta^{a}\left(f^{2}\left[\xi_{c}, \xi\right]\right)=0, \\
\theta\left(S\left(\xi_{c}, \xi\right)\right)=d \theta\left(\xi_{c}, \xi\right)=0,
\end{gathered}
$$

which reduce respectively to

$$
J_{d}^{a} \theta^{d}\left(\left[f \xi_{c}, \xi\right]\right)+\theta^{a}\left(\left[\xi_{c}, \xi\right]\right)=0,
$$

$$
d \theta \equiv 0 \quad\left(\bmod \theta^{c} \wedge \theta^{b}\right)
$$


because $\theta^{a} \circ f=J_{e}^{a} \theta^{e}$ and $J_{e}{ }^{a} J_{b}{ }^{e}=-\delta_{b}^{a}$. The equation (3.21) is equivalent to

$$
\phi_{c}{ }^{a}+J_{c}{ }^{e} J_{d}{ }^{a} \phi_{e}{ }^{a}=0, \quad \phi_{b}{ }^{a}=d \theta^{a}\left(\xi, \xi_{b}\right) .
$$

Thus (3.11), (3.22) and (3.23) imply (3.18) and (3.19), which prove Theorem 3.2.

Theorem 3.2 just proved was already proved by Kurita [4]. There are other interesting results closely related to Theorem 3.2 (See, for example, Hsu [3], Sasaki and Hsu [6]).

Let $\left\{\xi_{b}, \xi\right\}$ be a frame adapted to $D$ and $\left\{\theta^{a}, \theta\right\}$ be its dual coframe in a coordinate neighborhood $U$ of $M$. We now put*)

$$
\begin{array}{cc}
e_{\beta}=\frac{1}{\sqrt{2}}\left(\xi_{\beta}+\sqrt{-1} \xi_{n+\beta}\right), & e_{\bar{\beta}}=\frac{1}{\sqrt{2}}\left(\xi_{\beta}-\sqrt{-1} \xi_{n+\beta}\right), \quad e=\xi ; \\
\omega^{\alpha}=\frac{1}{\sqrt{2}}\left(\theta^{\alpha}-\sqrt{-1} \theta^{n+\alpha}\right), \quad \omega^{\bar{\alpha}}=\frac{1}{\sqrt{2}}\left(\theta^{\alpha}+\sqrt{-1} \theta^{n+\alpha}\right), \quad \omega=\theta .
\end{array}
$$

Then we have in $U$ a complex frame $\left\{e_{\beta}, e_{\bar{\beta}}, e\right\}$ adapted to $D$ and a complex coframe $\left\{\omega^{\alpha}, \omega^{\bar{\alpha}}, \omega\right\}$ dual to $\left\{e_{\beta}, e_{\bar{\beta}}, e\right\}$. The equation (3.11) stated in Theorem 3.1 reduce to

$$
\begin{aligned}
d \omega & =\sqrt{-1} G_{\gamma \bar{\beta}} \omega^{\gamma} \wedge \omega^{\bar{\beta}}+\omega \wedge \phi, \\
d \omega^{a} & =\phi_{\bar{\gamma} \beta}{ }^{a} \omega^{\bar{\gamma}} \wedge \omega^{\beta}+\omega \wedge \phi^{\alpha}
\end{aligned}
$$

with respect to a complex coframe adapted to $D$ such that $\omega=\theta$ and $\xi=e$. The equations (3.25) shows that the system of differential equations

$$
\omega=0, \quad \omega^{\alpha}=0 \quad(\alpha=1, \cdots, n)
$$

is completely integrable. That is to say, there is in $M$ a pseudo-conformal structure if $D$ is non-degenerate. Thus the hyperdistribution $D$ with complex structure determines a pseudo-conformal structure in $M$ if and only if $D$ is torsionless and non-degenerate. (See Cartan [1], Chern and Moser [2], Tanaka [7]). Thus we can state

THEOREM 3.3. Let $D$ be a hyperdistribution with complex structure in a manifold $M$. Then $D$ determines a pseudo-conformal structure in $M$ if it is torsionless and non-degenerate.

It is known that any real hypersurface $M$ immersed in a complex manifold admits a hyperdistribution $D$ with complex structure which is naturally induced in $M$ and that such a $D$ is always torsionless. Hence $M$ admits a pseudoconformal structure determined by $D$ if $D$ is non-degenerate (See Cartan [1], Chern and Moser [2], Tanaka [7], Yano and Ishihara [8]).

*) The indices $\alpha, \beta, \gamma, \delta, \varepsilon$ run over the range $\{1, \cdots, n\}$ and the summation convension will be used with respect to this system of indices. 
The equations (3.18) stated in Theorem 3.2 reduce to

$$
d \omega=\sqrt{\overline{-1}} G_{\gamma \bar{\beta}} \omega^{\gamma} \wedge \omega^{\bar{\beta}}, \quad d \omega^{\gamma}=\chi_{\beta}{ }^{\alpha} \wedge \omega^{\beta},
$$

where

$$
\chi_{\beta}^{\alpha}=\phi_{\bar{\gamma} \beta}^{\alpha} \omega^{\bar{\gamma}}+\phi_{\beta}^{\alpha} \omega
$$

with respect to a complex coframe $\left\{\omega^{\alpha}, \omega^{\bar{\alpha}}, \omega\right\}$ adapted to $D$ such that $\omega=\theta$ and $e=\xi$. The second equation of (3.26) shows that the system of differential equations

$$
\omega^{\alpha}=0 \quad(\alpha=1, \cdots, n)
$$

is completely integrable.

\section{§4. Pseudo-conf ormal mappings.}

Let $M$ and ' $M$ be manifolds admitting hyperdistributions $D$ and ' $D$ with complex structure respectively. Let $J$ and ' $J$ be the complex structures in $D$ and ' $D$ respectively. Assume there is a homeomorphism $\phi: M \rightarrow^{\prime} M$ such that, for any vector field $X$ being tangent to $M$ and belonging to $D, \phi_{*} X$ belongs to ${ }^{\prime} D$ and $\phi_{*} J={ }^{\prime} J \phi_{*}$ holds, where $\phi_{*}$ denotes the differential mapping of $\phi$. Then $\phi$ is called a pseudo-conformal mapping. Roughly speaking, $\phi$ is a pseudoconformal mapping if and only if $\phi$ leaves the hyperdistributions with complex structure invariant.

Let $\phi: M \rightarrow^{\prime} M$ be a pseudo-conformal mapping. Then, taking an almost contact structure $(f, \xi, \theta)$ associated with $D$ in a coordinate neighborhood $U$ of $M$, we see that $\bar{f}=\phi_{*} f\left(\phi_{*}\right)^{-1}, \quad \bar{\xi}=\phi_{*} \xi, \quad \bar{\theta}=\theta \circ\left(\phi_{*}\right)^{-1}$ form an almost contact structure $(\bar{f}, \bar{\xi}, \bar{\theta})$ associated with ${ }^{\prime} D$ in ${ }^{\prime} U=\phi(U)\left(\subset^{\prime} M\right)$. Thus, taking arbitrarily an almost contact structure $\left({ }^{\prime} f,{ }^{\prime} \xi,{ }^{\prime} \theta\right)$ associated with ${ }^{\prime} D$ in ${ }^{\prime} U$, we obtain in $' U$

$$
\bar{f}={ }^{\prime} f-^{\prime} \theta \otimes A, \quad \bar{\xi}=\frac{1}{\alpha}\left({ }^{\prime} \xi-{ }^{\prime} f A\right), \quad \bar{\theta}=\alpha^{\prime} \theta
$$

because of (2.2), where $\alpha$ is a non-vanishing function and $A$ a vector field belonging to ' $D$, both defined in ' $U$. Conversely, if a homeomorphism $\phi: M \rightarrow$ ' $M$ satisfies (4.1), then $\phi$ is a pseudo-conformal mapping. Thus we can state

THEOREM 4.1. The equation (4.1) is a necessary and sufficient condition for a homeomorphism $\phi: M \rightarrow ' M$ to be a pseudo-conformal mapping, where $M$ and ' $M$ are manifolds admitting respectively hyperdistributions $D$ and ' $D$ with complex structure.

We now have, using (2.6) and (4.1), 
Proposition 4.2. For a pseudo-conformal mapping $\phi: M \rightarrow^{\prime} M$

$$
G(X, Y)=\alpha^{\prime} G\left(\phi_{*} X, \phi_{*} Y\right)
$$

holds for any vector fields $X$ and $Y$ being tangent to $M$ and belonging to $D, \alpha$ being a non-vanishing function defined in ' $U$, where $G$ and ' $G$ are the Levi tensors of $D$ and ' $D$ respectively.

Taking account of Lemma 4.2, we have from (4.1)

Proposition 4.3. Let $M$ and ' $M$ be two manifolds with hyperdistributions $D$ and 'D with complex structure, respectively. Let there be given a pseudo-conformal mapping $\phi: M \rightarrow^{\prime} M$. Then ' $D$ is torsionless if and only if $D$ is so also.

When $M$ and ' $M$ are real hypersurfaces in a complex manifold $\tilde{M}$, they admit respectively hyperdistributions $D$ and ' $D$ with complex structure which are induced by their immersions from the complex structure of the ambient complex manifold $\tilde{M}$. If $\tilde{\phi}: \tilde{M} \rightarrow^{\prime} \tilde{M}$ is a holomorphic transformation of $\tilde{M}$ and $\tilde{\phi}(M)={ }^{\prime} M$, then the restriction $\phi: M \rightarrow^{\prime} M$ of $\tilde{\phi}$ to $M$ is a pseudo-conformal mapping (See Cartan [1], Chern and Moser [2], Tanaka [7], Yano and Ishihara [8]).

When a transformation $\phi: M \rightarrow M$ of a manifold $M$ admitting a hyperdistribution $D$ with complex structure is a pseudo-conformal mapping, $\phi$ is called a pseudo-conformal transformation of $M$. Let there be given a vector field $X$ in a manifold $M$ with hyperdistribution $D$ with complex structure and assume any local transformation $\phi_{t},(-\varepsilon<t<\varepsilon, \varepsilon>0)$ of $M$ generated by $X$ is always a pseudo-conformal transformation. Then $X$ is called an infinitesimal pseudoconformal transformation or, simply saying, a pseudo-conformal vector in $M$. Thus from Theorem 4.1 we have

THEOREM 4.4. A vector field $X$ in a mannfold $M$ admitting a hyperdistribution $D$ with complex structure is a pseudo-conformal vector if and only if for any almost contact structure $(f, \xi, \theta)$ associated with $D$ in a coordinate neighborhood $U$ of $M$

$$
L_{X} f=-\theta \otimes V, L_{X} \xi=-a \xi-f V, L_{X} \theta=a \theta
$$

are established, where $a$ is a function and $V$ a vector field belonging to $D$, i. e., $\theta(V)=0$, both defined in $U$.

Proposition 4.5. For any pseudo-conformal vector $X$ in $M$ admitting a hyperdistribution $D$ with complex structure

$$
L_{X} G \equiv a G \quad(\bmod \theta)
$$

is satısfied, where $G$ denotes the Levi tensor of $D$ and $a$ is a function appearing in (4.3). 
Proof. Denote by $G_{j i}$ components of $G$. Then, using (4.3), we have

$$
\begin{aligned}
& L_{X} G_{j i}= L_{X}\left[f_{\jmath}{ }^{k}\left(\partial_{k} \theta_{\imath}-\partial_{i} \theta_{k}\right)\right] \\
&=\left(L_{X} f_{\jmath}{ }^{k}\right)\left(\partial_{k} \theta_{\imath}-\partial_{i} \theta_{k}\right)+f_{\imath}{ }^{k} L_{X}\left(\partial_{k} \theta_{i}-\partial_{i} \theta_{k}\right) \\
&=-\left(\theta_{\jmath} V^{k}\right)\left(\partial_{k} \theta_{\imath}-\partial_{i} \theta_{k}\right)+a f_{\jmath}{ }^{k}\left(\partial_{k} \theta_{\imath}-\partial_{i} \theta_{k}\right) \\
& \quad+f_{\jmath}{ }^{k}\left(\partial_{k} a \theta_{\imath}-\partial_{\imath} a \theta_{k}\right),
\end{aligned}
$$

from which $L_{X} G \equiv a G,(\bmod \theta)$. This proves Proposition 4.5.

LEMMA 4.6. Let $X$ be a pseudo-conformal vector in a manifold $M$ admitting a hyperdistribution $D$ with complex structure. If $X$ belongs to $D$ then $G(Y, X)=0$ for any vector field $Y$ in $M$.

Proof. The last equation of (4.3) reduces to

$$
X^{j} \partial_{j} \theta_{k}+\partial_{k} X^{j} \theta_{j}=a \theta_{k},
$$

where $X=X^{i} \partial / \partial x^{i}$. Next, $\theta(X)=0$ implies $\partial_{k} X^{\jmath} \theta_{j}=-X^{j} \partial_{k} \theta_{\jmath}$. Thus we have

$$
X^{j}\left(\partial_{j} \theta_{k}-\partial_{k} \theta_{\jmath}\right)=a \theta_{k},
$$

from which, transvecting $f_{2}{ }^{k}$,

$$
X^{j}\left(\partial_{j} \theta_{k}-\partial_{k} \theta_{j}\right) f_{\imath}{ }^{k}=0 .
$$

This is equivalent to $G(Y, X)=0$.

As a corollary to Lemma 4.6, we have (See Tanaka [7])

THEOREM 4.7. Let $X$ be a pseudo-conformal vector in a manifold $M$ admitting a hyperdistribution $D$ with complex structure and assume that $D$ is torsionless and non-degenerate. Then, if $X$ belongs to $D, X$ vanishes identically.

When for an almost contact structure $(f, \xi, \theta)$ associated with a hyperdistribution $D$ with complex structure the vector field $\xi$ is pseudo-conformal, the structure $(f, \xi, \theta)$ is said to be regular. We now have

LEMMA 4. 8. An almost contact structure $(f, \xi, \theta)$ associated with $D$ is regular if and only if $P=0$ is satısfied.

Proof. Assume $(f, \xi, \theta)$ is regular. Then substituting $X=\xi$ into (4.3) gives $a=0$ and $V=0$ because of $L_{\xi} \xi=0$. Thus, substituting $a=0$ and $V=0$ into (4.3), we have $P=-L_{\xi} f=0$ and $Q=-L_{\hat{\xi}} \theta=0$. Conversely, if we assume $P=0$ for $(f, \xi, \theta)$, then we have $Q=0$ as a consequence of the proposition $\left(\mathrm{P}_{2}\right)$ stated in $\S 1$. Thus $L_{\xi} f=-P=0$ and $L_{\xi} \theta=-Q=0$, which are equivalent to the condition (4.3) with $a=0$ and $V=0$. Thus $(f, \xi, \theta)$ is regular. Hence Lemma 4.8 is proved. 
As a consequence of Lemmas 2.4 and 4.8., we have

THEOREM 4.9. Let $M$ be a manifold admitting a hyperdistribution $D$ with complex structure and assume $D$ is torsionless. Then an almost contact structure associated with $D$ is normal if and only if it is regular.

\section{BIBLIOGRAPHY}

[1] Cartan, E., Sur la géométrie pseudo-conforme des hypersurfaces des deux variables complexes I, Ann. Math. Pura Appl. (4) 11 (1932), 17-90 (or Oeuveres II, 2, 1231-1304) ; II, Ann. Scula Norm Pisa, (2) 1 (1932), 333-354 (or Oeuveres III, 2, 1217-1238).

[2] Chern, S.S. and J.K. Moser, Real hypersurfaces in complex manifolds, Acta Math., 133 (1974), 219-271'

[ 3 ] Hsu, C. J., Note on $(\phi, \xi, \eta)$-structure I, Tôhoku Math. J., 13 (1961), 434-442.

[4] Kurita, M., On normal contact metric manifolds, J. Math. Soc. Japan, 15 (1963), 304-318.

[5] SaSaki, S., Almost contact manifolds, Part I, Lecture Note, Tôhoku Unıv., Senda1 Japan, 1965.

[6] SASAKI, S. AND C. J. Hsu, On the integrability of almost contact structure, Tôhoku Math. J., 14 (1962), 167-176.

[7] TANAKA, N., On the pseudo-conformal geometry of hypersurfaces of the space of $n$ complex variables, J. Math. Soc. Japan, 14 (1962), 397-429.

[8] YANO, K. AND S. Ishinara, Real hypersurfaces of a complex manifolds and distributions with complex structure, Koda1 Math. J., 1 (1978), 289-303.

Tokyo Institute of Technology 